\title{
Could In-situ Analysis Help Unravel the Biochemical Milieu of Chronic Wounds?
}

\author{
Duncan Sharp* \\ Biomedicine Research group, Faculty of Health and Social Sciences, Leeds Beckett University, Leeds, LS1 3HE, UK
}

Received: September 09, 2014; Accepted: September 22, 2014; Published: October 13, 2014

*Corresponding author: Duncan Sharp, Biomedicine Research group, Faculty of Health and Social Sciences, Leeds Beckett University, Leeds, LS1 3HE, UK, Tel: +440-113-8129398; E-mail: d.sharp@leedsbeckett.ac.uk

The regulated series of events that occur in normal wounds (inflammation, proliferation and remodeling) act to rapidly restore skin integrity. However, failure to proceed normally through these stages results in wound chronicity - thereby remaining in a proinflammatory, proteolytic state [1]. The prolonged duration in the inflammatory phase results in excess inflammatory mediators, including pro-inflammatory cytokines, proteolytic agents (e.g. matrix metalloproteinases and serine proteinases) and reactive intermediates (e.g. reactive oxygen and nitrogen species) which can further perpetuate damage [2,3]. Several mechanisms have been proposed to explain this absence of complete wound healing, such as the 'fibrin cuff' theory, the 'inflammatory cell "trap"' theory and the involvement of cytokines, growth factors, reactive oxygen species, and proteolytic enzymes $[1,4]$. However, the complex biochemical processes that occur within chronic wounds are not comprehensively understood [4], and therefore, a better understanding of such processes may enable the design and implementation of new strategies to treat delayed healing more effectively [5].

Our understanding of the fundamental biochemical processes attributed to wound chronicity are inhibited by the limitation of suitable animal models, which cannot fully reflect the processes that occur during pathological wounds in humans [6], and the difficulties of modeling such events in-vitro [7]. As such, there has been great interest in the analysis of wound fluid for the identification and quantification of biomarkers to aid our understanding and seek suitable biomarkers for diagnostics. An expert consensus identified many potential biomarkers to enhance wound care including cytokines, enzymes and their substrates (e.g. matrix metalloproteinases), growth factors and hormones, inflammatory mediators, nitric oxide, reactive oxygen species, $\mathrm{pH}$ and nutritional factors (e.g. zinc or glutamine) [8]. However, the lack of specificity and selectivity in selecting and exploiting such biomarkers has been noted for many published studies [4]. Previous research has implicated shifts in $\mathrm{pH}$ and Reactive Oxygen Species (ROS) in the development and cellular disturbances that occur relating to chronicity. However, there are difficulties in their reliable analysis using conventional approaches and in-vitro methodologies due to the lack of suitable $\mathrm{pH}$ sensing technologies, the instability and reactivity of ROS species, and the transient localised fluctuation that may be anticipated for both $\mathrm{pH}$ and ROS. Therefore, the development and implementation of methodologies to allow the real-time insitu analysis of biomarkers, such as pH and ROS, may offer huge advantages in terms of our understanding of how the biomarkers change throughout normal and pathological healing processes, and provide a better insight to the factors initiating chronicity.

\section{pH: A key biomarker?}

The in-situ measurement of wound fluid pH is one of the most commonly studied biomarkers, largely reliant upon commercially available flat-bottomed $\mathrm{pH}$ probes which have shown that $\mathrm{pH}$ remains elevated in chronic wounds. Given the importance of $\mathrm{pH}$ to a range of biochemical processes, including the activity of proteolytic enzymes, the ability to monitor $\mathrm{pH}$ and modulate it (through treatments and dressings) could be advantageous. New technologies are emerging to enable monitoring of $\mathrm{pH}$ in wounds, based upon electrochemical methods using potentiometric and voltammetric methodologies [9-11], inductance-based pH responsive hydrogels [12], optical fiber based sensors [13], and luminescence approaches through sensor films [14]. Investigators have also tried to elucidate whether there is a spatial disparity to pH. Tsukada et al. [14] measured a pH gradient from most alkaline in the centre of chronic wounds to more acidic at the boundaries using a novel foil-sensor which offers potential to map surface $\mathrm{pH}$ within the wound. However, there are relatively few studies that have been designed to provide the requisite information to build a better understanding of the $\mathrm{pH}$ milieu in terms of longitudinal or periodical monitoring, and spatial resolution of $\mathrm{pH}$ throughout the normal and pathophysiological processes associated with chronicity. Hence, there is scope for new technologies to provide a better understanding of wound healing and the dynamic changes of $\mathrm{pH}$ in acute and chronic wounds [15].

\section{Reactive species and oxidative stress}

The roles of Reactive Oxygen Species (ROS) within wounds continues to diversify as more evidence is gained about the biochemistry of normal wounds and the pathophysiology of chronic wounds. The generation of ROS is essential for normal wound healing processes, including antimicrobial roles, cell signalling, wound debridement and clearance of apoptotic/ 
necrotic tissue during tissue remodeling [16]. However, whilst ROS are clearly important in normal cellular and biochemical processes, disturbances in the oxidant-antioxidant balance and exceeding the antioxidant capacity result in localised oxidative stress. The prolonged or elevated production of ROS is known to have consequences on many pathways [17] and the pathogenesis of chronic wounds [18]. Quantification of oxidative stress biomarkers may be useful to understand disease activity [19], however, the measurement of ROS directly in wound fluids (e.g. superoxide anion, hydroxyl radicals, singlet oxygen and hydrogen peroxide) are limited by their short lifetime and high reactivity. Therefore, the use of oxidative damage to proteins, DNA and lipids are widely used to indirectly study oxidative stress, using multiple indirect measures such as: total protein carbonyl content, malondialdehyde, 8-isoprostrane and total antioxidant capacity [20-22]. However, there is scope to explore the in-situ monitoring of individual ROS species, but also benefit to be derived from monitoring antioxidant species or antioxidant capacity, which may all provide information regarding the oxidant-antioxidant balance to aid in our understanding of the mechanisms relating to wound chronicity, Suzuki et al. [23] identified that being able to decipher the local and spatial coordination of ROS signals as a key future challenge. As knowledge of wound healing processes is elucidated further, the specific application of therapies can be enhanced and the exploration of multi-factorial treatments for different stages of healing can be sought to optimise wound healing [16,24].

To enable such monitoring, the interdisciplinary reapplication of technologies from more established in-vivo systems could be explored. A range of methodologies have been used to quantify the rapid fluctuations in ROS in-vivo, such as using fluorescence, chemiluminescence and electroanalytical platforms, exemplar approaches include: superoxide anion radical monitoring for circulatory and brain tissue investigations [25,26], bioluminescence reporters (e.g. PCL-1) to monitor hydrogen peroxide fluxes [27], chemiluminescence probes (e.g. L-012) to image ROS and Reactive Nitrogen Species (RNS) [28], or luminol for in-vivo chemiluminescence imaging of ROS [29]. The translation of such approaches into wound science could offer huge advantages, and as such, knowledge and expertise may be drawn from in-vivo ROS monitoring,

\section{Interdiscipliniarity in the future and SOJ Biochemistry}

Within the plethora of potential biomarkers that have been identified as associated with wound healing problems, a focus towards the spatial localisation, real-time monitoring, and an ability to monitor wound biochemistry in-situ could offer huge advantages to our understand of healing and the mechanisms driving pathophysiological processes associated with chronicity.

The specific markers of $\mathrm{pH}$ and ROS offer the advantages of the potential for re-application of emerging technologies, or through technologies that are currently under development directly for this purpose. Whilst the real-time in-situ monitoring of such biomarkers is challenging, there is huge potential to advance our understanding of the fundamental biochemistry of normal and pathophysiological processes, and to better our knowledge of the mechanisms underlying treatments and dressing.

To enhance such areas of research, improved interdisciplinary communication can help bridge between these different areas which is where the interdisciplinary nature of SOJ Biochemistry can facilitate this dissemination for a breadth of research.

\section{References}

1. Eming SA, Krieg T, Davidson JM. Inflammation in wound repair: molecular and cellular mechanisms. J invest dermatol. 2007; 127(3): 514-25.

2. Moseley R, Stewart JE, Stephens P, Waddington RJ, Thomas DW. Extracellular matrix metabolites as potential biomarkers of disease activity in wound fluid: lessons learned from other inflammatory diseases? Br J Dermatol. 2004; 150(3): 401-413.

3. Schneider LA, Korber A, Grabbe S, Dissemond J. Influence of $\mathrm{pH}$ on wound-healing: a new perspective for wound-therapy? Arch of Dermatol Res. 2007; 298(9): 413-420.

4. Liu YC, Margolis DJ, Isseroff RR. Does Inflammation Have a Role in the Pathogenesis of Venous Ulcers? A Critical Review of the Evidence. J Invest Dermatol. 2011; 131(4): 818-827. doi: 10.1038/jid.2010.428.

5. Diegelmann RF, Evans MC. Wound healing: An overview of acute, fibrotic and delayed healing. Front Biosci. 2004; 9: 283-289.

6. Lindblad WJ. Animal models in wound healing research: do we need more? Wound Repair Regeneration. 2000; 8(2): 81-82.

7. Mustoe TA, O'Shaughnessy K, Kloeters O. Chronic wound pathogenesis and current treatment strategies: a unifying hypothesis. Plast Reconstr surg. 2006; 117(7 Suppl): 35S-41S.

8. (WUWHS).Diagnostics and wounds. A consensus document. Principles of best practice: 2008 .

9. Phair J, Newton L, McCormac C, Cardosi MF, Leslie R, Davis J. A disposable sensor for point of care wound $\mathrm{pH}$ monitoring. Analyst. 2011; 136(22): 4692-4695. doi: 10.1039/c1an15675f.

10.Sharp D. Printed composite electrodes for in-situ wound $\mathrm{pH}$ monitoring. Biosens Bioelectron. 2013; 50: 399-405. doi: 10.1016/j. bios.2013.06.042.

11.Schroter A, Walther A, Fritzsche K, Kothe J, Wolff AR, Gerlach G. Infection monitoring in wounds. 2nd International Conference on Bio-Sensing Technology. 2012; 6: 175-183. doi:10.1016/j. proche.2012.10.144.

12. Sridhar V, Takahata K. A hydrogel-based passive wireless sensor using a flex-circuit inductive transducer. Sens Actuators A Phys. 2009; 155(1): 58-65.

13. Schyrr B, Paschec S, Scolana E, Ischera R, Ferrarioa D, Porchet JA, et al. Development of a polymer optical fiber $\mathrm{pH}$ sensor for on-body monitoring application. SENSOR ACTUAT B-CHEM. 2014; 194: 238248.

14. Schreml S, Meier RJ, Kirschbaum M, Kong SC, Gehmert S, Felthaus 0 , et al. Luminescent dual sensors reveal extracellular pH-gradients and hypoxia on chronic wounds that disrupt epidermal repair. Theranostics. 2014; 4(7): 721-35. doi: 10.7150/thno.9052.

15. Schreml S, Szeimies RM, Karrer S, Heinlin J, Landthaler M, Babilas P. The impact of the $\mathrm{pH}$ value on skin integrity and cutaneous wound healing. J Eur Acad Dermatol Venereol. 2010; 24(4): 373-378. doi: 10.1111/j.1468-3083.2009.03413.x. 
16. Bryan N, Ahswin H, Smart N, Bayon Y, Wohlert S, Hunt JA. Reactive oxygen species (ROS)--a family of fate deciding molecules pivotal in constructive inflammation and wound healing. Eur Cell Mater. 2012; 24: 249-65

17. Martindale JL, Holbrook NJ. Cellular response to oxidative stress: Signaling for suicide and survival. J Cell Physiol. 2002; 192(1): 1-15

18. Wlaschek M, Scharffetter-Kochanek K. Oxidative stress in chronic venous leg ulcers. Wound Repair Regen. 2005. 13(5): 452-461.

19. James TJ, Hughes MA, Cherry GW, Taylor RP. Evidence of oxidative stress in chronic venous ulcers. Wound Repair Regen. 2003; 11(3): 172-176.

20. Moseley R, Hilton JR, Waddington RJ, Harding KG, Stephens P, Thomas DW. Comparison of oxidative stress biomarker profiles between acute and chronic wound environments. Wound Repair Regen. 2004; 12(4) 419-29.

21. McInnes RL, Cullen BM, Hill KE, Price PE, Harding KG, Thomas DW, et al. Contrasting host immuno-inflammatory responses to bacterial challenge within venous and diabetic ulcers. Wound Repair Regen 2014; 22(1): 58-69. doi: 10.1111/wrr.12133.

22. Yeoh-Ellerton S, Stacey MC. Iron and 8-isoprostane levels in acute and chronic wounds. J Invest Dermatol. 2003; 121(4): 918-25.

23. Suzuki N, Mittler R. Reactive oxygen species-dependent wound responses in animals and plants. Free Radic Biol Med. 2012; 53(12) 2269-76. doi: 10.1016/j.freeradbiomed.2012.10.538.

24. Kapoor M, Appleton I. Wound healing: Abnormalities and future therapeutic targets. Curr Anaesth Crit Care. 2005; 16(2): 88-93. doi:10.1016/j.cacc.2005.03.005

25. Fujita M, Tsuruta R, Kasaoka S, Fujimoto K, Tanaka R, Oda Y, et al. In vivo real-time measurement of superoxide anion radical with a novel electrochemical sensor. Free Radic Biol Med. 2009; 47(7): 1039-48. doi:10.1016/j.freeradbiomed.2009.07.012.

26. Fabian RH, DeWitt DS, Kent TA. In vivo detection of superoxide anion production by the brain using a cytochrome c electrode. J Cereb Blood Flow Metab. 1995; 15(2): 242-247.

27.Van de Bittner GC, Dubikovskaya EA, Bertozzi CR, Chang CJ. In vivo imaging of hydrogen peroxide production in a murine tumor model with a chemoselective bioluminescent reporter. Proc Natl Acad Sci U S A. 2010; 107(50): 21316-21. doi: 10.1073/pnas.1012864107.

28. Kielland A, Blom T, Nandakumar KS, Holmdahl R, Blomhoff R, Carlsen $\mathrm{H}$. In vivo imaging of reactive oxygen and nitrogen species in inflammation using the luminescent probe L-012. Free Radic Biol Med. 2009; 47(6): p. 760-766. doi: 10.1016/j.freeradbiomed.2009.06.013.

29. Chen WT, Tung $\mathrm{CH}$, Weissleder R. Imaging reactive oxygen species in arthritis. Mol Imaging. 2004; 3(3): 159-62. 